\title{
Add-On Treatment with Pregabalin for Patients with Uncontrolled Neuropathic Pain Who Have Been Referred to Pain Clinics
}

\author{
José-Luis de la Calle · José De Andres • \\ María Pérez • Vanessa López
}

Published online: 22 October 2014

(C) The Author(s) 2014. This article is published with open access at Springerlink.com

\begin{abstract}
Objective The aim of this study was to investigate the impact of pregabalin on pain, other symptoms, and patientreported outcomes for patients with uncontrolled pain who have been referred to pain clinics.

Patients and Methods Adult patients with uncontrolled pain who had a score of $\geq 4$ in the DN4 questionnaire were evaluated at baseline, month 3, and month 6 . Evaluations included pain levels using a visual analog (VAS) scale as well as anxiety, depression, sleep, disability, and treatment satisfaction employing validated tools.

Results Our sample comprised 413 patients who met the selection criteria, had not received pregabalin previously, and were prescribed pregabalin at the study initiation, mainly (97\%) as add-on therapy. Overall, patients had a statistically significant reduction in VAS pain score of 41 points (54\% reduction, $p<0.001$ ), varying from $64 \%$ reduction (oncological pain) to $31 \%$ reduction (central neuropathic pain). Effect sizes for anxiety, depression, sleep, and treatment satisfaction improvement were moderate to large depending on the dimension and clinical entity. Conclusion Our results suggest that in patients with uncontrolled neuropathic pain of various origins who were
\end{abstract}

J.-L. de la Calle

Pain Unit, Hospital Ramón y Cajal, Madrid, Spain

J. De Andres

Anesthesia, Critical Care, and Multidisciplinary Pain

Management Department, Valencia University Medical School,

Valencia University General Hospital, Valencia, Spain

M. Pérez $(\bowtie)$ · V. López

Medical Unit, Pfizer España, Avda. de Europa, 20 B, Parque Empresarial La Moraleja, Alcobendas, 28108 Madrid, Spain e-mail: maria.perez2@pfizer.com treated at pain clinics, the addition of pregabalin to a wider pharmacological treatment regimen was associated with a clinically relevant improvement of pain and psychological well-being and a reduction in the impact of neuropathic pain on daily activities. Add-on treatment with pregabalin was well tolerated.

\section{Key Points}

Refractory or uncontrolled neuropathic pain is common and is associated with significant burden.

Our results suggest that in patients with uncontrolled neuropathic pain of various origins who were treated at pain clinics, treatment with pregabalin as part of a wider pharmacological regimen is associated with a significant improvement of pain, an improvement of anxiety and depressive symptoms, a reduction in sleep disturbance, and an amelioration of the interference of the disease with the patient's life.

\section{Introduction}

The management of neuropathic pain requires an interdisciplinary approach in which pharmacological treatment is cardinal [1]. Although the epidemiologic information on refractory neuropathic pain is limited [2], there is a general agreement that many patients with neuropathic pain are refractory or unable to tolerate existing treatments $[3,4]$. In fact, several surveys carried out in Europe and the US have shown that although patients with neuropathic pain receive several drugs for pain treatment, moderate to severe levels 
of pain are common and are associated with a significant burden in terms of reduced quality of life, loss of productivity, and increased use of health resources [5-8].

Pregabalin is an $\alpha 2-\delta$ ligand that, in randomized controlled trials, has been demonstrated to be effective for the treatment of several peripheral or central neuropathic painful conditions such as diabetic neuropathy, postherpetic neuralgia, trigeminal neuralgia, and the central neuropathic pain associated with spinal cord injury, among others [9]. Several non-randomized studies have evaluated the impact of pregabalin for the treatment of refractory neuropathic pain [10-18]; however, these studies were carried out in a primary care setting $[10,11,13,14]$, were focused on specific clinical entities [10, 12, 17], specifically evaluated patients refractory to gabapentin [13, 14], and/or used an economic model for the evaluation $[15,16]$. In addition, patients with neuropathic pain, especially those with refractory pain, are commonly treated by combining two or more drugs [19]. Although there are some randomized trials on the use of pregabalin combined with other pharmacological treatment in patients with neuropathic pain [20-23], none addressed the treatment of refractory pain. It has been suggested that the treatment of neuropathic pain should be aimed at the mechanism underlying the specific type of pain [24]. The effect of pregabalin on the different types of neuropathic pain (i.e. the different types of spontaneous or evoked pain) has scarcely been investigated.

The aim of this report was to investigate the impact of pregabalin on pain, other symptoms, and patient-reported outcomes for patients with uncontrolled pain who were referred to pain clinics. We also aimed to explore the results of pregabalin treatment in this population according to the underlying clinical entity and type of pain.

\section{Patients and Methods}

The UNIDOL study was an observational, multicenter, prospective study performed by 161 investigators from pain clinics throughout Spain between February 2009 and February 2010 with the aim of evaluating the clinical profile and previous management of patients with uncontrolled neuropathic pain who were referred to pain clinics. The study was approved by the Ethics Committee of the Hospital General Universitario de Valencia (Spain). Written informed consent was obtained from every subject. The study was carried out in accordance with the principles contained in the Declaration of Helsinki.

To be included in the UNIDOL study, patients had to be 18 years of age or older, have been referred to a pain clinic with uncontrolled pain, and have a score equal to or greater than 4 in the DN4 questionnaire. Patients were excluded if they were unable to understand the study objectives or fulfill the study self-administered questionnaires. Results for the whole cohort (i.e. 728 evaluable patients) presenting data on the clinical profile and previous management of patients with uncontrolled neuropathic pain who were referred to pain clinics have been accepted for publication elsewhere [25]. We present herein the results of an analysis of the subset of patients from the UNIDOL cohort who had not received pregabalin previously and were prescribed this drug upon entry to the study $(n=413)$.

\subsection{Study Assessments}

At baseline, the following information was recorded: sociodemographic data, type of specialist referring the patient, diagnostic confirmation of neuropathic pain, confirmation of the presence of uncontrolled pain as judged by the investigator, etiology and duration of pain, causes for uncontrolled pain, and pain intensity as measured with a 0-100 mm visual analog scale (VAS). Investigators were asked to record the presence and severity of the several types of evoked or spontaneous neuropathic pain based on the physical examination and/or clinical interview. We also recorded pharmacological and non-pharmacological treatment for neuropathic pain. In addition, the Spanish validated versions of the following questionnaires and scales were fulfilled: DN4 questionnaire, Hospital Anxiety and Depression Scale (HADS), Medical Outcomes Study Sleep Scale (MOS-Sleep), the World Health Organization Disability Assessment Schedule (WHO-DAS II), and the Treatment Satisfaction with Medicines Questionnaire (SATMED-Q). Clinical evaluations were also administered at months 3 and 6 .

The neuropathic pain diagnostic questionnaire DN4 consists of 10 items describing different pain characteristics. A score of at least 4 of 10 possible points is considered to identify neuropathic pain with $83 \%$ sensitivity and $90 \%$ specificity [26-28].

The HADS is a self-administered instrument consisting of 14 items: seven items exploring depression symptoms, and the other seven exploring anxiety symptoms [29, 30]. Each item score ranges from 0 to 3 , where 0 represents the absence of that symptom and 3 represents the highest severity or frequency of the symptom. By adding the seven items of each subscale, two scores ranging from 0 to 21 are obtained for depression and anxiety (HADS-D and HADSA, respectively).

The MOS-Sleep is also a self-administered questionnaire evaluating key aspects of sleep [31, 32]. It consists of 12 items composing six subscales or domains: sleep disturbances, snoring, shortness of breath or headache upon awakening, adequacy of sleep, day somnolence, and amount of sleep. In addition, the MOS-Sleep provides a 
summary index of sleep disturbances that can be obtained from nine of its item scores; the higher the score, the worse the sleep, with the exception of amount of sleep and adequacy of sleep dimensions, which are scored in the opposite direction. In patients with neuropathic pain, this scale has shown adequate psychometric properties [32].

The WHO-DAS II comprises 12 items that evaluate an individual's level of functioning and disability in six areas: understanding and communicating, getting around, selfcare, getting along with people, life activities, and participation in society [33-35]. Patients are required to answer questions regarding how many difficulties they had in the last 30 days due to their health condition, on a 5-point scale from 1 (none) to 5 (extreme difficulty or cannot do it). Raw scores are transformed into a standard scale ranging from 0 to 100 , with higher scores reflecting greater disability. A global score is obtained that ranges from 0 to 700 (if work activities outside the home are assessed) or from 0 to 600 .

The SATMED-Q is a self-administered questionnaire involving 17 items that evaluate six dimensions: treatment effectiveness, convenience of use, impact on daily activities, medical care, global satisfaction, and undesirable side effects [36, 37]. Regarding the side effects, the SATMED$Q$ records information about whether the patient has experienced side effects with the medication, and contains three questions addressing how the side effects interfere with physical activity, leisure and free-time activities, and daily activities. It also provides a global score for satisfaction with drug treatment by summing up the scores of all domains. Raw scores are transformed into a scale ranging from 0 to 100 , with greater scores indicating higher satisfaction.

\subsection{Statistical Analysis}

Quantitative variables were described using means and standard deviations (SDs) and qualitative variables with absolute and relative frequencies. The changes from baseline to month 6 in the previously-mentioned scales and subscales scores, including the pain intensity VAS, were used as outcome variables. In addition, the percentage of patients with a reduction of at least $50 \%$ in pain intensity, as rated by VAS pain, was calculated, and these patients were defined as responders.

The significances of within-group changes from baseline to endpoint in the total scores or subscores of the scales and questionnaires were calculated using Student's $t$-test or a non-parametric test; for binary outcomes, we used the McNemar test. Effect sizes for these within-group changes were also calculated using the difference of means, before and after treatment, of a particular measure, divided by the SD of that measure before treatment [38]. For effect size interpretation, the established criterion of considering 0.20 to $<0.50$ to be a small effect size, $\geq 0.50$ to $<0.80$ to be moderate, and $\geq 0.80$ to be large was used [38].

\section{Results}

\subsection{Patient Disposition and Demographic and Clinical Characteristics}

Originally, 755 patients were included in the UNIDOL cohort. We excluded 27 (3.6\%) patients who did not meet the selection criteria, leaving 728 evaluable patients in the cohort. From those patients, 413 patients had not received pregabalin previously, were prescribed pregabalin at the study initiation, and constituted the sample for these analyses and subsequent report. Overall, 34 of the 413 patients $(8.2 \%)$ discontinued their participation in the study.

Patients' demographic and clinical characteristics are described in Table 1. Patients had a mean age of 58 years and were predominantly women (62\%). Patients had severe pain with a mean VAS score of 76, a mean duration of pain over 2 years, and a mean DN4 score of 6.6. The most common cause of neuropathic pain was radiculopathy (44\%), followed by pain of oncological origin [i.e. malignant-, radiation-, or chemotherapy-induced pain] (14\%) and neuralgia ( $9 \%$ ). With the exception of thermal allodynia, reported in $64 \%$ of the patients, each type of spontaneous or evoked pain was reported by at least $75 \%$ of the patients.

There were no differences in pain severity or duration of pain among the several clinical entities included in this analysis (data not shown). However, the mean DN4 score differed significantly among these clinical entities $(p<0.001)$, with patients with central neuropathic pain (7.7), diabetic neuropathy (7.6), complex regional syndrome (7.5), and other neuropathies (7.0) showing the highest scores on this scale. The remaining clinical entities have the following DN4 scores: oncological pain (6.9), trigeminal neuralgia (6.6), post-surgery/trauma (6.4), other neuralgia (6.4), radiculopathy (6.3), nerve entrapment syndrome (6.2), and plexopathy (5.7).

\subsection{Treatment Characteristics}

Treatment characteristics are described in Table 2. The vast majority of patients $(97 \%)$ received pregabalin as add-on therapy. The mean $( \pm S D)$ final dose of pregabalin was $319 \pm 375 \mathrm{mg} /$ day in patients receiving pregabalin add-on and $209 \pm 114 \mathrm{mg} /$ day in those receiving pregabalin monotherapy. In patients receiving pregabalin add-on, the dose varied greatly depending on the clinical entity; patients with plexopathy (585 $\pm 664 \mathrm{mg} /$ day) and those 
Table 1 Demographic and clinical characteristics

\begin{tabular}{|c|c|c|}
\hline Characteristic & $N$ & Value \\
\hline Age [years; mean $\pm \mathrm{SD}$ ] & 393 & $57.93 \pm 13.83$ \\
\hline Sex [female; $n(\%)]$ & 402 & $249(61.9)$ \\
\hline BMI $\left[\mathrm{kg} / \mathrm{m}^{2}\right]$ & 357 & \\
\hline Mean \pm SD & & $26.72 \pm 4.41$ \\
\hline DN4 (0-10) $[$ mean \pm SD] & 413 & $6.55 \pm 1.54$ \\
\hline VAS $[$ mean \pm SD $]$ & 398 & $75.55 \pm 14.94$ \\
\hline Duration of pain [years; mean $\pm \mathrm{SD}$ ] & 408 & $2.26 \pm 3.40$ \\
\hline Causes of neuropathic pain $[n(\%)]$ & 392 & \\
\hline Neuropathy & & $22(5.6)$ \\
\hline Neuralgia & & $35(8.9)$ \\
\hline Radiculopathy & & $171(43.6)$ \\
\hline Nerve entrapment syndrome & & $27(6.9)$ \\
\hline Plexopathy & & $16(4.1)$ \\
\hline Complex regional pain syndrome & & $17(4.3)$ \\
\hline Post-surgery/trauma & & $32(8.2)$ \\
\hline $\begin{array}{l}\text { Malignant/radiation/chemotherapy-induced } \\
\text { pain }\end{array}$ & & $53(13.5)$ \\
\hline Central neuropathic pain & & $10(2.6)$ \\
\hline Other neuropathic pain & & $9(2.3)$ \\
\hline Spontaneous pain $[n(\%)]$ & 387 & \\
\hline Lancinating & & $354(91.5)$ \\
\hline Burning & & $353(89.8)$ \\
\hline Paresthesia & & $370(94.4)$ \\
\hline Dysesthesia & & 367 (94.6) \\
\hline Evoked pain $[n(\%)]$ & 385 & \\
\hline Static allodynia & & $300(77.9)$ \\
\hline Dynamic allodynia & & $308(79.6)$ \\
\hline Thermal allodynia & & $239(63.9)$ \\
\hline Mechanical hyperalgesia & & $322(82.1)$ \\
\hline Hyperpathia & & $281(74.1)$ \\
\hline
\end{tabular}

$B M I$ body mass index, $S D$ standard deviation, $V A S$ visual analog scale

with trigeminal neuralgia ( $576 \pm 452 \mathrm{mg} /$ day) received the higher doses, whereas patients with radiculopathy received the lower doses $(242 \pm 244 \mathrm{mg} /$ day $)$. Almost three-quarters of patients received at least four drugs for the treatment of neuropathic pain, with the most common being opioids (61\%), antidepressants $(50 \%)$ and, to a much lesser extent, non-steroidal anti-inflammatories $(23 \%)$. The doses of the most commonly prescribed drugs are presented in Table 3. Two-thirds of the patients were also receiving non-pharmacological interventions.

\subsection{Treatment Outcomes for the Overall Sample and by Clinical Entity}

Overall, patients treated with add-on pregabalin showed a statistically significant reduction of VAS pain score of 41 points (54\% reduction), from a baseline mean score of 76 points to an endpoint mean score of 35 (Table 4). Patients with oncological pain (64\% reduction), complex regional pain syndrome (61\% reduction), and trigeminal neuralgia (59\% reduction) had the largest reductions in VAS pain score, whereas patients with central neuropathic pain showed the lowest reduction (31\% reduction). The effect sizes for these changes were large for all clinical entities, with the exception of central neuropathic pain, which was moderate. At study completion, $79 \%$ of patients showed a reduction of pain intensity of at least $50 \%$ from baseline (i.e. responders). The proportion of responders was higher among those with oncological pain $(88 \%)$ and complex regional pain syndrome $(87 \%)$, and lower among patients with central neuropathic pain $(50 \%)$ and patients with diabetic neuropathy $(58 \%)$.

There were no differences in the improvement of pain according to the type of pain (spontaneous or evoked) [Table 5]. In fact, the proportion of responders (VAS pain reduction $\geq 30 \%$ ) varies from $87 \%$ in patients with paresthesias to $89.4 \%$ in patients with mechanical hyperalgesia.

Significant and clinically relevant improvements (i.e. effect sizes moderate) were observed in all other dimensions of the disease (Table 4). The percentage reduction over the 6 months of pregabalin treatment was $39 \%$ for anxiety, $35 \%$ for depression, and $38 \%$ for sleep disorders. The proportion of patients with bad or very bad health according to the WHO-DAS II was reduced from $51.4 \%$ at baseline to $9.2 \%$ at month 6 . Similarly, the proportion of patients with severe or extreme interference with life was reduced from $44 \%$ to $14 \%$ during the same period. Treatment satisfaction, as evaluated with the SATMED-Q total score, increased $50 \%$ compared with the baseline score. Regarding improvement of these dimensions of the disease by clinical entity, the results followed a similar pattern to that previously mentioned for pain: patients with complex regional syndrome, plexopathy, and trigeminal neuralgia exhibited better results, whereas lesser improvements were reported in patients with central neuropathic pain and in patients with diabetic neuropathy and other neuropathy. Effect sizes for anxiety, depression, sleep, and treatment satisfaction improvement were moderate to large depending on the dimension and clinical entity (Table 4). Despite effect sizes for most dimensions in patients with central neuropathic pain being only moderate, the effect size for the treatment satisfaction was large.

\subsection{Tolerability}

The proportion of patients experiencing side effects according to the question included in the SATMED-Q significantly increased from $51 \%$ at baseline to $65 \%$ at 


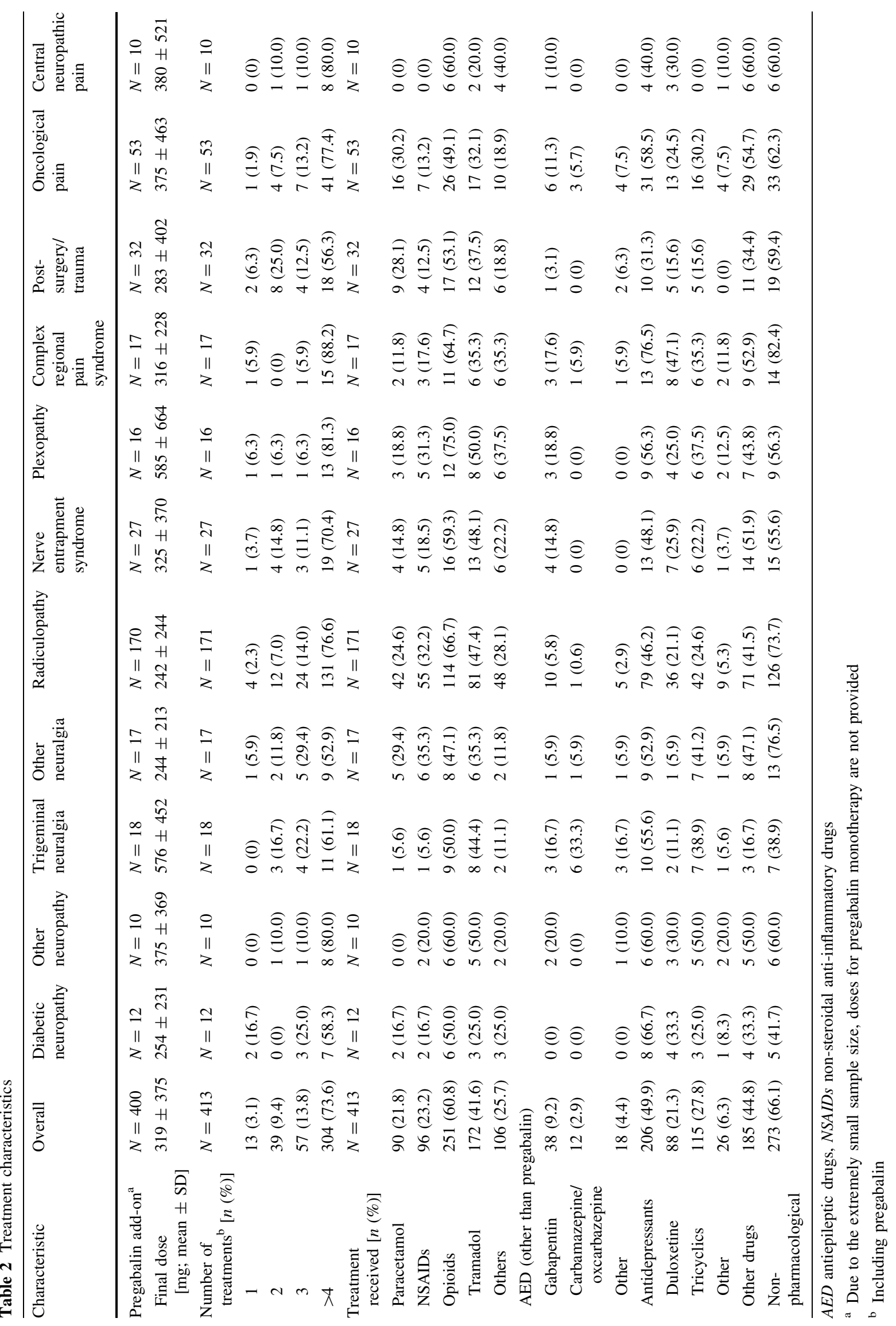


Table 3 Doses of the most commonly $(N \geq 10)$ prescribed drugs with pregabalin
$A E D$ antiepileptic drugs, $N$ number of patients who received the drug, $n$ number of patients with available information about the dose, NSAIDs non-steroidal antiinflammatory drugs, $S D$ standard deviation

\begin{tabular}{lccc}
\hline Drug & $N$ & $n$ & Dose [mean \pm SD] \\
\hline Paracetamol (mg/day) & 90 & 86 & $2,576.8 \pm 921.9$ \\
NSAIDs (mg/day) & & & \\
$\quad$ Ibuprofen & 39 & 38 & $1,194.74 \pm 456.16$ \\
Metamizole & 35 & 32 & $2,204.13 \pm 1,207.85$ \\
Diclofenac & 12 & 12 & $106.25 \pm 37.12$ \\
Dexketeprofen & 10 & 10 & $65.63 \pm 18.60$ \\
Opioids & & & \\
Tramadol (mg/day) & 172 & 169 & $207.54 \pm 113.59$ \\
Fentanyl ( $\mu \mathrm{g} / \mathrm{h})$ & 65 & 62 & $18.60 \pm 15.61$ \\
Oxycodone (mg/day) & 96 & 93 & $34.19 \pm 29.57$ \\
Hydromorphone hydrochloride (mg/day) & 17 & 17 & $8.24 \pm 8.54$ \\
AED [other than pregabalin] (mg/day) & & & \\
Gabapentin & 38 & 38 & $1,334.7 \pm 660.4$ \\
Carbamazepine/oxcarbazepine & 12 & 12 & $650 \pm 416.7$ \\
Antidepressants (mg/day) & & & \\
$\quad$ Duloxetine & 88 & 87 & $32.19 \pm 18.56$ \\
Amitriptyline & 121 & 121 & \\
\hline
\end{tabular}

month 3 ( $p<0.001$ for the comparison with baseline) and $58 \%$ at month $6(p<0.05)$. The overall interference of side effects with patient's activities, as measured with the corresponding dimension of the SATMED-Q, increased slightly but significantly from a score of 75.5 at baseline to a score of 81.3 at endpoint (i.e. a $7.7 \%$ increase).

\section{Discussion}

Our results suggest that, in patients with uncontrolled neuropathic pain of various origins who were treated at pain clinics, treatment with pregabalin as part of a wider pharmacological regimen is associated with a relevant and significant improvement of pain, an improvement of anxiety and depressive symptoms, a reduction in sleep disturbance, and an amelioration of the interference of the disease with the patient's life.

Pregabalin in combination with other drugs produced a substantial improvement in pain, i.e. a $54 \%$ reduction in pain severity. In fact, almost $80 \%$ of the patients were considered responders (i.e. had a reduction of at least $50 \%$ in the VAS pain score). Previous studies with pregabalin in patients categorized as refractory have shown good but more modest results. In an analysis of 81 patients who had moderate to severe neuropathic pain despite treatment with gabapentin, a tricyclic antidepressant, and a third drug, Stacey and coworkers reported a mean improvement of $34 \%$ in the VAS score of the Short-Form McGill Pain Questionnaire, and a proportion of responders of $39 \%$ and $35 \%$ after 3 and 15 months, respectively, of pregabalin treatment [39]. However, these authors use a selected sample of patients who had previously participated in double-blind, placebo-controlled trials of pregabalin in patients with diabetic peripheral neuropathy or postherpetic neuralgia. The results from observational studies of shortterm treatment with pregabalin show a proportion of responders of $43 \%$ in 174 patients with neuropathic pain who were refractory to gabapentin and were treated in primary care environments $(34 \%$ in patients who received pregabalin add-on) [14], $54 \%$ in 244 patients with refractory neck pain treated in orthopedic surgery and rehabilitation clinics [12], and $62 \%$ in 564 patients with refractory low-back pain treated in orthopedic surgery and rehabilitation clinics [17]. Although the differences in the design, outcome measures and, in particular, underlying disease may account for the differences between our results and the results of these studies, we believe that they cannot entirely explain such a large difference. The lack of a control group could have overestimated the treatment effects in our study; however, the same criticism could be applied to all previous studies. In our view, a plausible explanation is the setting in which the patients were treated. Patients in the present study were treated at pain clinics, and therefore it is likely that, in this setting, treatment was optimized to a greater extent than in patients treated by a primary care physician or other specialists. Interestingly, the mean dose of pregabalin in the present study (319 mg/day) was higher than in the above-mentioned studies, $222 \mathrm{mg} /$ day in the study involving primary care [14], and $190 \mathrm{mg} /$ day in the study of patients with refractory low-back pain attended to by specialists [17]. This finding supports our hypothesis of greater treatment optimization at the pain clinics compared with other 


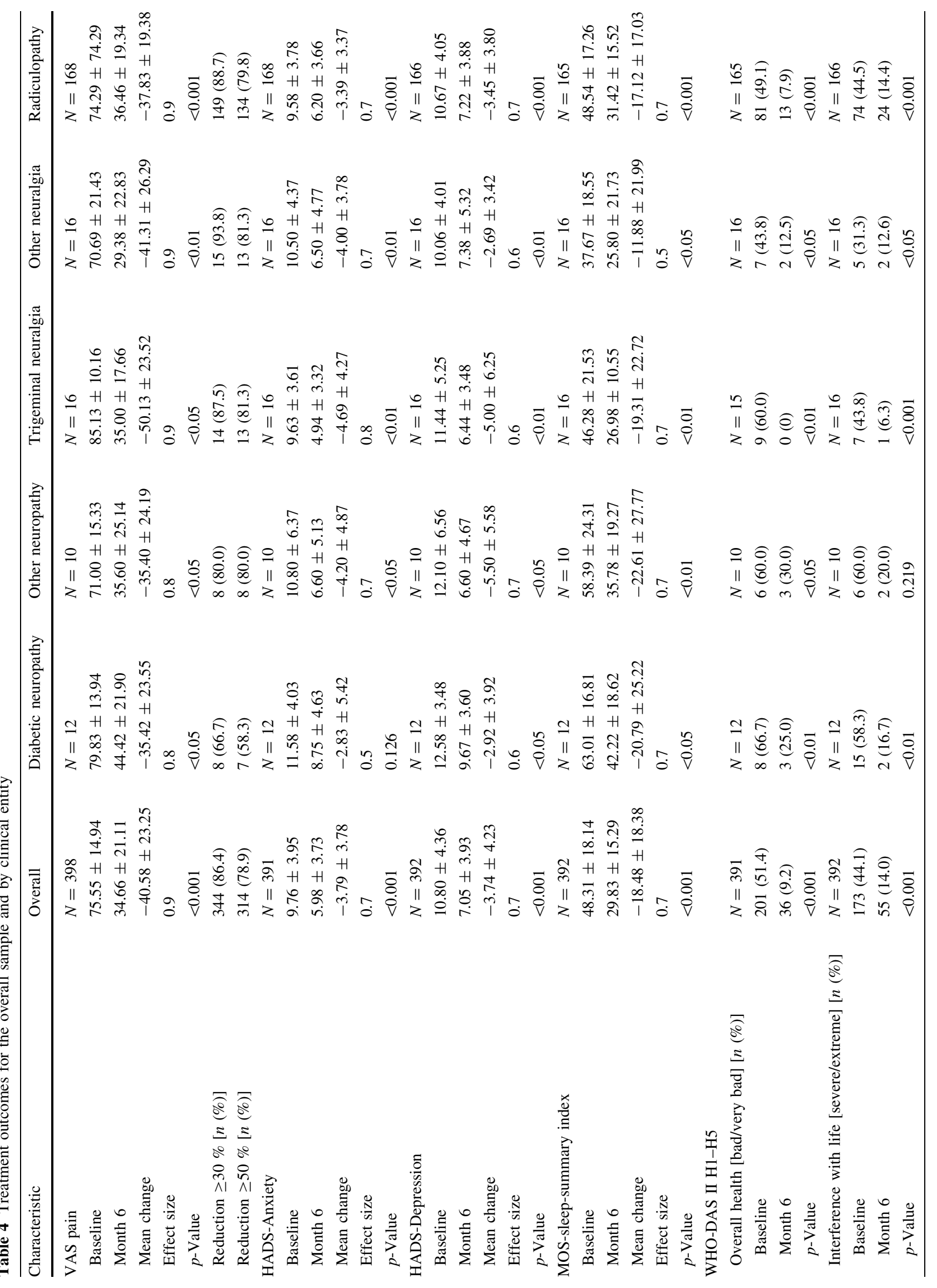




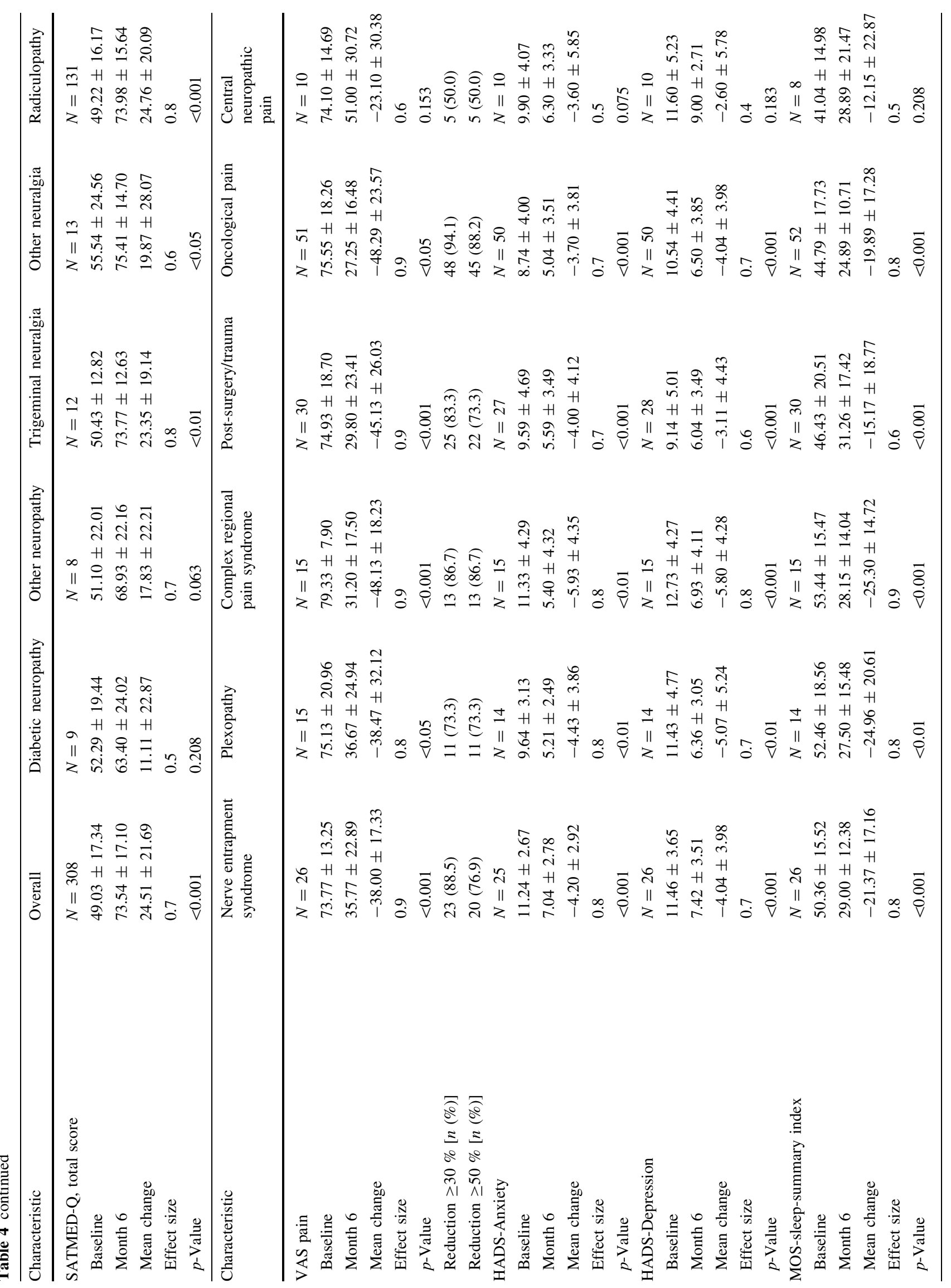




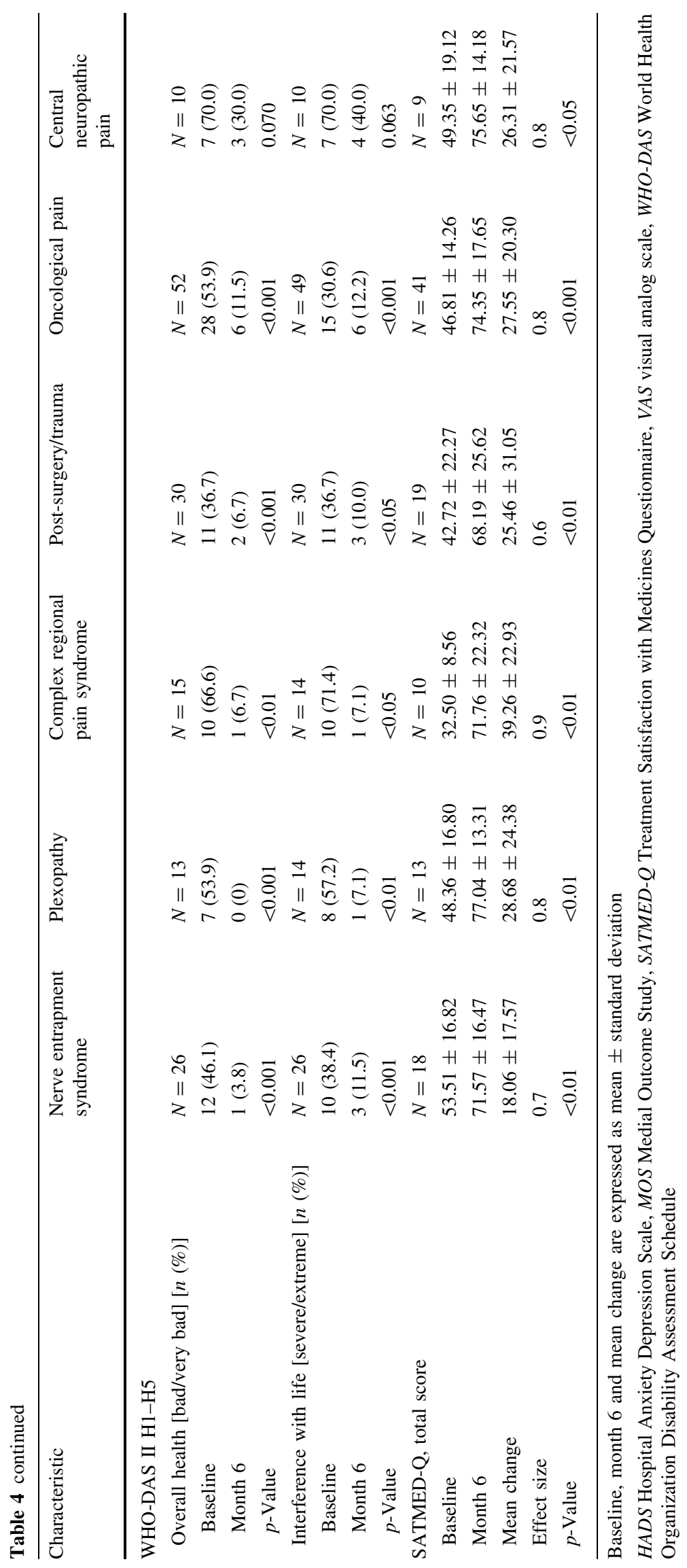




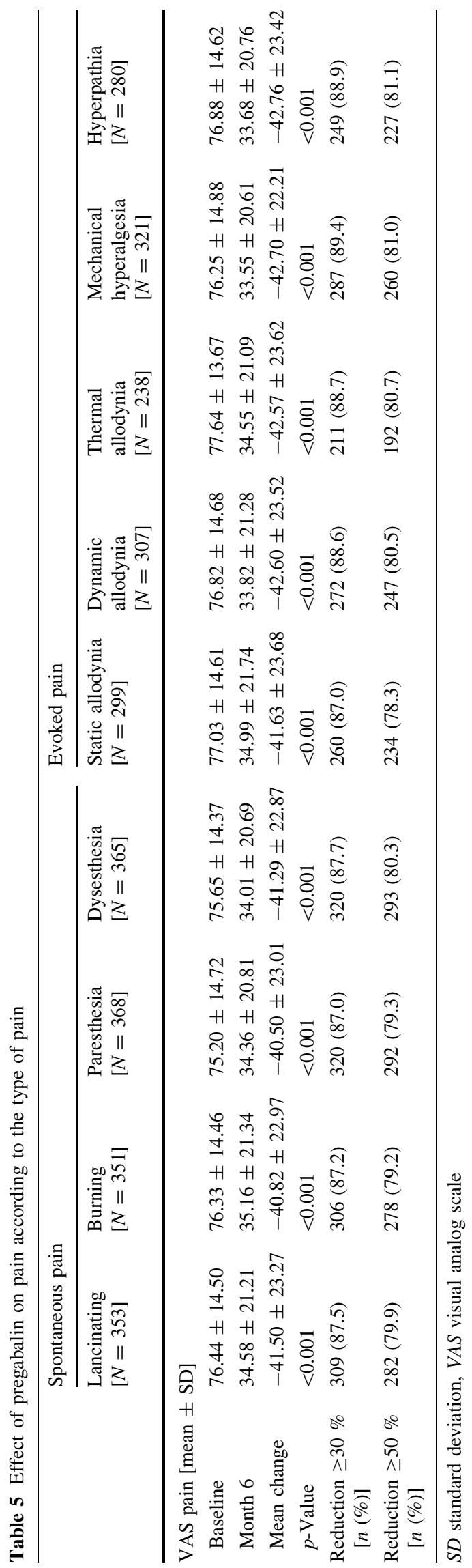

settings as the reason for the better results in the present study than in previous studies.

The results from other secondary efficacy outcomes are consistent with, and support, those obtained on pain. Relevant improvements in anxiety, depression, subjective sleep quality, and quality of life were observed after treatment with add-on pregabalin. This improvement in other dimensions of neuropathic pain is consistent with what it is known from randomized clinical trials in patients with neuropathic pain [9] and from previous studies on patients with refractory neuropathic pain $[12$, $14,17,40]$.

Although the results should be interpreted cautiously due to the nature of the analyses, the lack of a control group, and the small sample size for some subgroups, our analyses revealed that add-on pregabalin was similarly effective across all clinical entities studied. Patients with diabetic neuropathy, particularly those with central neuropathic pain, exhibited more modest results, with reductions in pain severity of $44 \%$ and $31 \%$, respectively, and response rates of $58 \%$ and $50 \%$, respectively. As we will comment below, these subgroups of patients had a higher score on the DN4 questionnaire. Whether this higher score reflects a worse clinical condition or a greater neuropathic component of the pain is impossible to answer. Pregabalin has consistently been demonstrated, in several randomized clinical trials, to be effective for the treatment of painful diabetic neuropathy, and in fact is considered a first-line option for the treatment of this condition in most clinical guidelines [9]. Central neuropathic pain is very disabling and difficult to treat, and only a few drugs in a few studies have shown positive results for this condition [9]. Pregabalin has been shown to be significantly more effective than placebo in three of the four studies carried out in patients with central neuropathic pain [9], including a recent trial in patients with neuropathic pain secondary to spinal cord injury [41]. However, whereas the response rates in these studies were $22 \%$ and $35 \%$, the response rates in randomized trials of pregabalin in painful diabetic neuropathy were usually over $45 \%$ [9]. Our results in patients with central neuropathic pain are consistent with this latter observation, indicating that although pregabalin is useful for the treatment of these patients, we must expect more modest benefits when treating patients with central neuropathic pain than in other conditions. The effect of pregabalin was almost identical across the several types of spontaneous or evoked pain; however, this subgroup analysis was difficult to interpret because most patients presented most types of pain.

The tolerability of pregabalin, as evaluated with the SATMED-Q, was good. Although there was an increased impact from side effects on the patients' lives, the effect was small (i.e. a $7 \%$ increase) and was offset by the 
benefits of the drug because treatment satisfaction increased by $50 \%$ at the endpoint.

In addition to the limitations mentioned, another potential limitation of our study was whether the patients included had truly refractory neuropathic pain. This issue is difficult to solve because despite recent initiatives [42], there is no standard definition for refractoriness. A recent survey has shown that non-optimized treatment is more frequent than truly treatment-refractory neuropathic pain in the community setting [43]. However, regardless of whether they are labeled as having refractory or uncontrolled pain, several factors appear to indicate that the patients included in this study were suffering disabling and very difficult-totreat neuropathic pain. Almost half of the patients reported having bad or very bad health and that the interference of their health condition with their lives was severe or extreme. More importantly, for pain management, the patients required a complex pharmacological regimen that included an opioid in almost two-thirds of the patients. Finally, it is important to stress that patients received pregabalin as part of a more complex pharmacological regimen; therefore, the attributable benefit to pregabalin in this population of patients with uncontrolled pain can only be elucidated in a randomized controlled trial.

It interesting to note that after initiating add-on pregabalin, the clinical entities with poorer results on pain amelioration and other secondary efficacy outcomes were central neuropathic pain and diabetic neuropathy, and these entities were those presenting the higher baseline score in the DN4 questionnaire. It is therefore possible that the use of the DN4 questionnaire could help to screen the presence of refractory neuropathic pain, a possibility that is worth evaluating in further psychometric studies of this assessment tool.

\section{Conclusions}

Despite the limitations of this study, these results suggest that, in the clinical practice setting, the addition of pregabalin to the previous treatment might be a useful alternative for the treatment of uncontrolled neuropathic pain. It would be worthwhile to carry out randomized clinical trials of pregabalin in patients with refractory pain that could confirm our positive results. The use of active comparators could help to delineate the role of pregabalin for treating refractory neuropathic pain.

Acknowledgments The authors thank Fernando Rico-Villademoros from COCIENTE S.L. for his assistance in the preparation of the draft of this manuscript; this assistance was funded by Pfizer, S.L.U.

Conflicts of interest Dr. De Andres has no conflicts of interest relevant to this manuscript; Dr. De la Calle has received consulting/ lecture fees from Boston Scientific, Medtronic, Grunenthal, Prostrakan, Mundipharma, Eisai, Janssen-Cilag, Cardiva and Pfizer; Dra. Pérez and Vanessa López are employees of Pfizer.

Open Access This article is distributed under the terms of the Creative Commons Attribution Noncommercial License which permits any noncommercial use, distribution, and reproduction in any medium, provided the original author(s) and the source are credited.

\section{References}

1. Carter GT, Galer BS. Advances in the management of neuropathic pain. Phys Med Rehabil Clin N Am. 2001;12:447-59.

2. Taylor RS. Epidemiology of refractory neuropathic pain. Pain Pract. 2006;6:22-6.

3. National Institute for Health and Clinical Excellence. Neuropathic pain: the pharmacological management of neuropathic pain in adults in non-specialist settings. In NICE clinical guideline 96. London: National Institute for Health and Clinical Excellence; 2010.

4. Dworkin RH, O'Connor AB, Audette J, et al. Recommendations for the pharmacological management of neuropathic pain: an overview and literature update. Mayo Clin Proc. 2010;85:S3-14.

5. Gore M, Brandenburg NA, Hoffman DL, et al. Burden of illness in painful diabetic peripheral neuropathy: the patients' perspectives. J Pain. 2006; 7:892-900.

6. Perez C, Saldana MT, Navarro A, et al. Prevalence and characterization of neuropathic pain in a primary-care setting in Spain: a cross-sectional, multicentre, observational study. Clin Drug Investig. 2009;29:441-50.

7. Tolle T, Dukes E, Sadosky A. Patient burden of trigeminal neuralgia: results from a cross-sectional survey of health state impairment and treatment patterns in six European countries. Pain Pract. 2006;6:153-60.

8. McDermott AM, Toelle TR, Rowbotham DJ, et al. The burden of neuropathic pain: results from a cross-sectional survey. Eur $\mathrm{J}$ Pain. 2006;10:127-35.

9. Pérez C, Margarit C, Gálvez R. A review of pregabalin for the treatment of peripheral and central neuropathic pain and its place in the treatment of chronic pain. Clin Med Rev Ther. 2011; 11:325-46.

10. de Salas-Cansado M, Perez C, Saldana MT, et al. An economic evaluation of pregabalin versus usual care in the management of community-treated patients with refractory painful diabetic peripheral neuropathy in primary care settings. Prim Care Diabetes. 2012;6:303-12.

11. de Salas-Cansado M, Perez C, Saldana MT, et al. A cost-effectiveness analysis of the effect of pregabalin versus usual care in the treatment of refractory neuropathic pain in routine medical practice in Spain. Pain Med. 2012;13:699-710.

12. Florez-Garcia M, Ceberio-Balda F, Morera-Dominguez C, et al. Effect of pregabalin in the treatment of refractory neck pain: cost and clinical evidence from medical practice in orthopedic surgery and rehabilitation clinics. Pain Pract. 2011;11:369-80.

13. Navarro A, Saldana MT, Perez C, et al. Costs and health resources utilization following switching to pregabalin in individuals with gabapentin-refractory neuropathic pain: a post hoc analysis. Pain Pract. 2012;12:382-93.

14. Saldana MT, Perez C, Navarro A, et al. Pain alleviation and patient-reported health outcomes following switching to pregabalin in individuals with gabapentin-refractory neuropathic pain in routine medical practice. Clin Drug Investig. 2012;32:401-12. 
15. Gordon J, Lister S, Prettyjohns M, et al. A cost-utility study of the use of pregabalin in treatment-refractory neuropathic pain. J Med Econ. 2012;15:207-18.

16. Prettyjohns M, Sandelin R, Lister S, Norrefalk JR. A cost-utility study of the use of pregabalin added to usual care in refractory neuropathic pain patients in a Swedish setting. J Med Econ. 2012;15:1097-109.

17. Morera-Dominguez C, Ceberio-Balda F, Florez-Garcia M, et al. A cost-consequence analysis of pregabalin versus usual care in the symptomatic treatment of refractory low back pain: subanalysis of observational trial data from orthopaedic surgery and rehabilitation clinics. Clin Drug Investig. 2010;30:517-31.

18. Lampl C, Schweiger C, Haider B, Lechner A. Pregabalin as mono- or add-on therapy for patients with refractory chronic neuropathic pain: a post-marketing prescription-event monitoring study. J Neurol. 2010;257:1265-73.

19. Harden N, Cohen M. Unmet needs in the management of neuropathic pain. J Pain Symptom Manage. 2003;25:S12-7.

20. Zin CS, Nissen LM, O'Callaghan JP, et al. A randomized, controlled trial of oxycodone versus placebo in patients with postherpetic neuralgia and painful diabetic neuropathy treated with pregabalin. J Pain. 2010;11:462-71.

21. Romano CL, Romano D, Bonora C, Mineo G. Pregabalin, celecoxib, and their combination for treatment of chronic low-back pain. J Orthop Traumatol. 2009;10:185-91.

22. Achar A, Chatterjee G, Ray TG, Naskar B. Comparative study of clinical efficacy with amitriptyline, pregabalin, and amitriptyline plus pregabalin combination in postherpetic neuralgia. Indian $\mathbf{J}$ Dermatol Venereol Leprol. 2010;76:63-5.

23. Rehm S, Binder A, Baron R. Post-herpetic neuralgia: $5 \%$ lidocaine medicated plaster, pregabalin, or a combination of both? A randomized, open, clinical effectiveness study. Curr Med Res Opin. 2010;26:1607-19.

24. Woolf CJ, Mannion RJ. Neuropathic pain: aetiology, symptoms, mechanisms, and management. Lancet. 1999;353:1959-64.

25. de Andres J, de la Calle JL, Perez M, Lopez V. Clinical characteristics, patient-reported outcomes, and previous therapeutic management of patients with uncontrolled neuropathic pain referred to pain clinics. Pain Res Treat. 2014;2014:518716.

26. Bouhassira D, Attal N, Alchaar H, et al. Comparison of pain syndromes associated with nervous or somatic lesions and development of a new neuropathic pain diagnostic questionnaire (DN4). Pain. 2005;114:29-36.

27. Bouhassira D, Attal N, Fermanian J, et al. Development and validation of the neuropathic pain symptom inventory. Pain. 2004;108:248-57.

28. Perez C, Galvez R, Huelbes S, et al. Validity and reliability of the Spanish version of the DN4 (Douleur Neuropathique 4 questions) questionnaire for differential diagnosis of pain syndromes associated to a neuropathic or somatic component. Health Qual Life Outcomes. 2007;5:66.
29. Zigmond AS, Snaith RP. The hospital anxiety and depression scale. Acta Psychiatr Scand. 1983;67:361-70.

30. Herrero MJ, Blanch J, Peri JM, et al. A validation study of the hospital anxiety and depression scale (HADS) in a Spanish population. Gen Hosp Psychiatry. 2003;25:277-83.

31. Hays RD, Stewart AL. Sleep measures. In: Stewart AL, Ware JE (eds). Measuring functioning and well-being: the Medical Outcomes Study approach. Durham, NC: Duke University Press; 1992. pp. 235-259.

32. Rejas J, Ribera MV, Ruiz M, Masrramon X. Psychometric properties of the MOS (Medical Outcomes Study) Sleep Scale in patients with neuropathic pain. Eur J Pain. 2007;11:329-40.

33. World Health Organization. World Health Organization Disability Assessment Schedule II. 12-item self-administered version. Geneva: World Health Organization; 2004.

34. Luciano JV, Ayuso-Mateos JL, Fernandez A, et al. Psychometric properties of the twelve item World Health Organization Disability Assessment Schedule II (WHO-DAS II) in Spanish primary care patients with a first major depressive episode. J Affect Disord. 2010;121:52-8.

35. Vazquez-Barquero JL, Vazquez Bourgon E, Herrera Castanedo S, et al. Spanish version of the new World Health Organization Disability Assessment Schedule II (WHO-DAS-II): initial phase of development and pilot study. Cantabria disability work group. Actas Esp Psiquiatr. 2000;28:77-87.

36. Rejas J, Ruiz MA, Pardo A, Soto J. Minimally important difference of the Treatment Satisfaction with Medicines Questionnaire (SATMED-Q). BMC Med Res Methodol. 2011;11:142.

37. Ruiz MA, Pardo A, Rejas J, et al. Development and validation of the "Treatment Satisfaction with Medicines Questionnaire" (SATMED-Q). Value Health. 2008;11:913-26.

38. Kazis LE, Anderson JJ, Meenan RF. Effect sizes for interpreting changes in health status. Med Care. 1989;27:S178-89.

39. Stacey BR, Dworkin RH, Murphy K, et al. Pregabalin in the treatment of refractory neuropathic pain: results of a 15-month open-label trial. Pain Med. 2008;9:1202-8.

40. Plested M, Budhia S, Gabriel Z. Pregabalin, the lidocaine plaster and duloxetine in patients with refractory neuropathic pain: a systematic review. BMC Neurol. 2010;10:116.

41. Cardenas DD, Nieshoff EC, Suda K, et al. A randomized trial of pregabalin in patients with neuropathic pain due to spinal cord injury. Neurology. 2013;80:533-9.

42. Smith BH, Torrance N, Ferguson JA, et al. Towards a definition of refractory neuropathic pain for epidemiological research. An international Delphi survey of experts. BMC Neurol. 2012;12:29.

43. Torrance N, Ferguson JA, Afolabi E, et al. Neuropathic pain in the community: more under-treated than refractory? Pain. 2013;154:690-9. 